\begin{tabular}{|c|l|}
\hline Title & Conductance bistability of gold nanowires at room temperature \\
\hline Author(s) & Kiguchi, Manabu; Konishi, Tatsuya; Murakoshi, Kei \\
\hline Citation & $\begin{array}{l}\text { Physical Review B, 73(12), 125406-1-125406-5 } \\
\text { https://doi.org/10.1103/PhysRevB.73.125406 }\end{array}$ \\
\hline Issue Date & 2006-03 \\
\hline Doc URL & http://hdl.handle.net/2115/8453 \\
\hline Rights & Copyright $\odot 2006$ A merican Physical Society \\
\hline Type & article \\
\hline File Information & PhysRevB73_p125406.pdf \\
\hline
\end{tabular}

Instructions for use 


\title{
Conductance bistability of gold nanowires at room temperature
}

\author{
Manabu Kiguchi, ${ }^{1}$ Tatsuya Konishi, ${ }^{1}$ and Kei Murakoshi ${ }^{1,2}$ \\ ${ }^{1}$ Department of Chemistry, Graduate School of Science, Hokkaido University, Sapporo, Hokkaido 060-0810, Japan \\ ${ }^{2}$ Precursory Research for Embryonic Science and Technology (PRESTO), Japan Science and Technology Agency, Sapporo, \\ Hokkaido 060-0810, Japan \\ (Received 10 January 2006; published 9 March 2006)
}

\begin{abstract}
Quantized conductance behavior of gold nanowires was studied under electrochemical potential control. We fabricated 1-nm-long monoatomic wires in solution at room temperature. Electrochemical potential significantly affected the stability of the monoatomic wire and fractional conductance peak occurrence in the conductance histogram. We revealed that the hydrogen adsorption on gold monoatomic wires was a decisive factor of the fractional peak, which was originated from the dynamic structural transition between two bistable states of the monoatomic wire showing the unit and the fractional values of the conductance. We could tune the stability of these bistable states to make the fractional conductance state preferable.
\end{abstract}

DOI: 10.1103/PhysRevB.73.125406

PACS number(s): 73.63.Rt, 73.40.Cg, 73.40.Jn

\section{INTRODUCTION}

Fabrication of stable atomic scale materials with quantized properties at room temperature is a central issue of material science. As one of the promising systems, gold nanowires have attracted wide attention. The conductance of the gold nanowire is often clearly quantized in the units of $G_{0}\left(1 G_{0}=2 e^{2} / h=12.9 \mathrm{k} \Omega^{-1}\right) .{ }^{1,2}$ Successful preparations of a monoatomic gold wire have been reported with a scanning tunneling microscope (STM) or a mechanically controllable break junction (MCBJ). ${ }^{3,4}$ While quantized conductance behavior of the gold nanowire with the ideal unit of $G_{0}$ seems to be well established in certain cases, the appearance of abnormal fractional conductance in the gold nanowire has recently attracted much attention. ${ }^{5-15}$ Several models such as spin polarized conductance, ${ }^{5,6}$ structural deformation, ${ }^{7-10}$ scattering on impurities and defects, ${ }^{11-13}$ and change in the electronic states ${ }^{14,15}$ have been proposed to explain the behavior of fractional conductance. These models assume that the origins of fractional conductance are deviation of the one-dimensional (1D) monoatomic wire from the ideal structure and the possible contribution of Peierls transition. ${ }^{7}$ If one can assume the formation of the gold monoatomic wire, the structural transition between bistable states with distinct values of conductance, e.g., Peierls transition, can also contribute to well-defined fractional values at the conductance quantization. Although the origin of the fractional conductance has not been clarified yet, it seems to be very important to control the quantized behavior of $1 \mathrm{D}$ materials by external perturbation, especially for the control of the reversible transition between the two states $\left(1 G_{0}\right.$ and fractional conductance).

While there are several external perturbations, such as temperature, pressure, electric and magnetic fields, etc., we paid attention to the electrochemical potential. In contrast to other external perturbations, the electrochemical potential can tune precisely the absolute potential energy of electrons in the nanowire. Recently, successful observations of conductance quantization at nanowires under electrochemical potential control have been reported for $\mathrm{Au}, \mathrm{Ag}, \mathrm{Pb}, \mathrm{Pd}, \mathrm{Pt}$,
$\mathrm{Ni}$, etc. ${ }^{15-19}$ Fractional conductance was also observed by Xuo et al. for the gold nanowire in aqueous solution. ${ }^{15}$ However, the origin of the fractional conductance has not been clarified yet.

To evaluate the origin of the fractional conductance observed in the gold nanowire, it is necessary to clarify the decisive factors. The measurement of dynamic changes in conductance is also important. In the present study, we studied conductance quantization of the gold nanowire at room temperature under electrochemical potential control. The stability of the nanowire with fractional conductance was successfully controlled by the electrochemical potential. The origin of the fractional value was discussed based on the analysis of the time-dependent change in the conductance values at the stretched process of the nanowires.

\section{EXPERIMENTAL}

The experimental design used in this study was the same as described in detail in our previous report. ${ }^{19}$ The experiments were performed in a four-electrode electrochemical cell mounted in a chamber that was filled with high purity $\mathrm{N}_{2}$ gas to reduce the effect of oxygen. The gold nanowire was created by driving a STM tip in and out of contact with a gold substrate at a typical rate of $50 \mathrm{~nm} / \mathrm{s}$ in the electrochemical cell. The tip was made of a gold wire (diameter $0.25 \mathrm{~mm}$ ) coated with wax to eliminate ionic conduction. The gold substrate was $\mathrm{Au}(111)$ prepared by the frame annealing and quenching method. The electrochemical potential $(\phi)$ of the gold substrate and the tip was controlled using a potentiostat (Pico-Stat, Molecular Imaging Co.) with a $\mathrm{Ag} / \mathrm{AgCl}$ reference electrode. A $0.50 \mathrm{~mm}$ diameter Pt wire was used as a counter electrode. The electrolyte was $0.1 \mathrm{M}$ $\mathrm{Na}_{2} \mathrm{SO}_{4}$ or $50 \mathrm{mM} \mathrm{H} \mathrm{HO}_{4}$. Conductance of the gold nanowire was calculated on the basis of the observed current between the tip and the substrate at the potential difference of $20 \mathrm{mV}$.

\section{RESULTS AND DISCUSSION}

Figure 1 shows the typical conductance traces and the conductance histogram of gold nanowires observed at $\phi=$ 

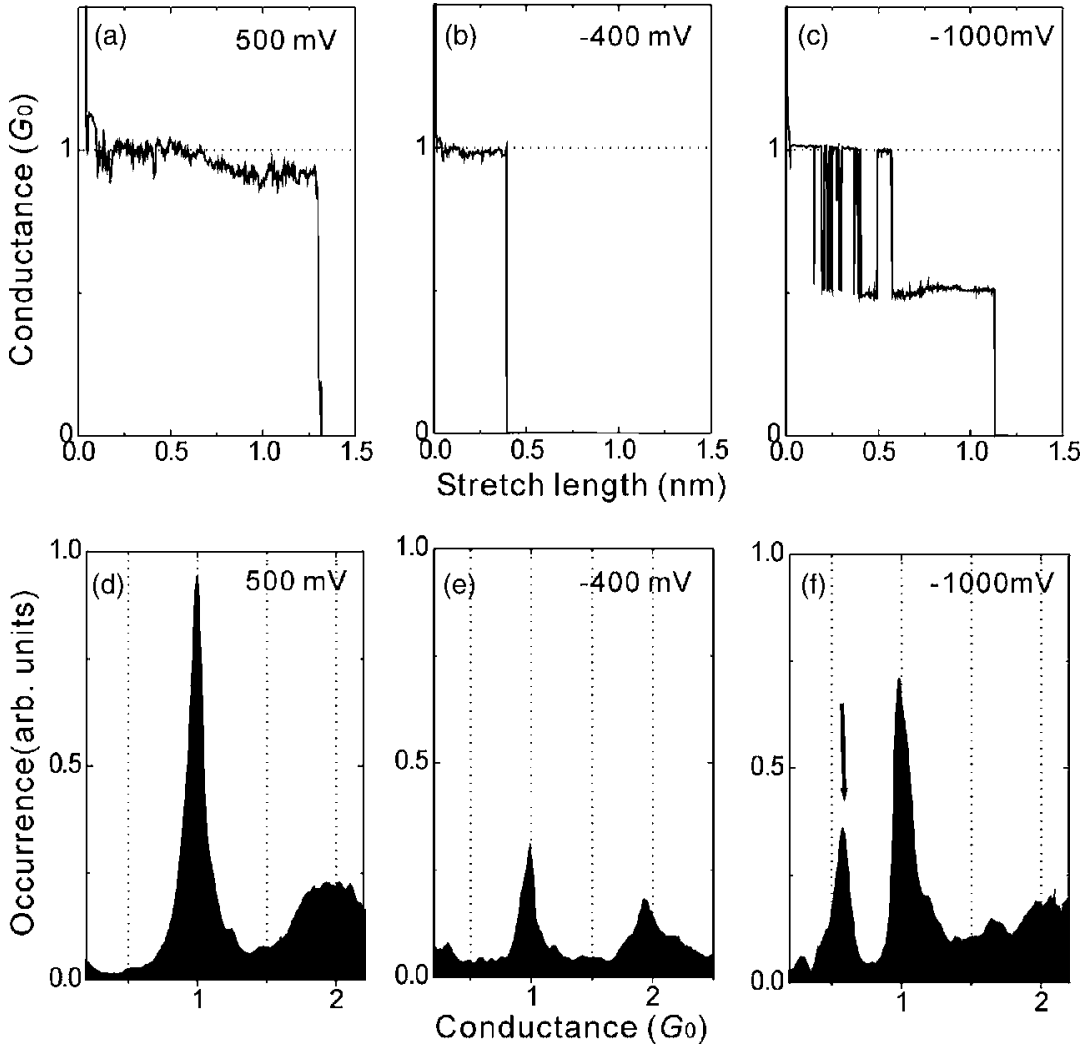

FIG. 1. (a)-(c) Conductance trace and (d)-(f) conductance histogram of gold nanowires in $0.1 \mathrm{M} \mathrm{Na}_{2} \mathrm{SO}_{4}$ at electrochemical potential of (a),(d) $+500 \mathrm{mV}$, (b),(e) $-400 \mathrm{mV}$, and (c),(f) $-1000 \mathrm{mV}$.
$+500,-400$, and $-1000 \mathrm{mV}$ in $0.1 \mathrm{M} \mathrm{Na}_{2} \mathrm{SO}_{4}$. Each conductance histogram was obtained for a large number (over 3000) of individual conductance traces. The plateau of $1 G_{0}$ stretched $1 \mathrm{~nm}$ in length at $+500 \mathrm{mV}$ [Fig. 1(a)]. Figure 2 shows the distribution of lengths for the last conductance plateau for Au. The length of the last plateau was defined as the distance between the points at which the conductance dropped below $1.3 G_{0}$ and $0.7 G_{0}$, respectively. The experimental results (dot in the figure) could be fitted with four distinct Gaussian functions. The values of the Gaussian peaks located at $0.00,0.22,0.48$, and $0.75 \mathrm{~nm}$, respectively. The interpeak distances were about $0.25 \mathrm{~nm}$, that were in agreement with the calculated $\mathrm{Au}-\mathrm{Au}$ distance in the gold monoatomic wire. ${ }^{1}$ Since it has been shown that the conductance of the monoatomic gold contact is $1 G_{0}, 1,2$ the $1-\mathrm{nm}$

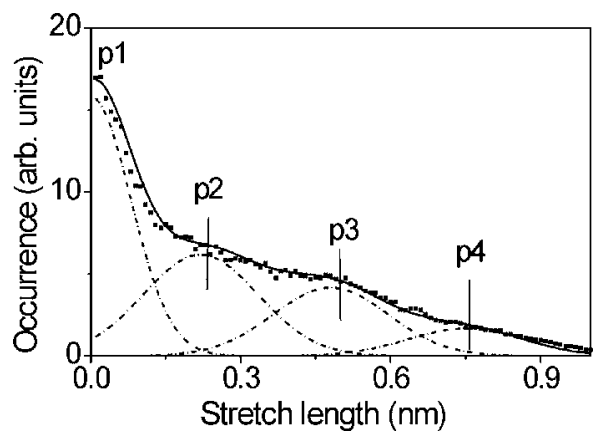

FIG. 2. The distribution of lengths for the last conductance plateau for Au. The length of the last plateau was defined as the distance between the points at which the conductance dropped below $1.3 G_{0}$ and $0.7 G_{0}$, respectively. The dot: experimental data, line: fitting results with distinct four Gaussian functions (dotted line). long $1 G_{0}$ plateau in Fig. 1 and the length histogram in Fig. 2 proved the formation of the gold monoatomic wire in the present system. While a 2-nm-long gold monoatomic wire is fabricated at $4.2 \mathrm{~K}$ in ultrahigh vacuum (UHV), ${ }^{4,20}$ there are few reports of the formation of a monoatomic wire at room temperature. The present results proved that the electrochemical method made it possible to fabricate a stable gold monoatomic wire at room temperature in solution.

The length of the conductance plateau was dependent upon the electrochemical potential. Figure 3 shows the averaged stretch length of the gold monoatomic wire as a function of $\phi$. As the potential was scanned from $500 \mathrm{mV}$ to negative, the length decreased at $200 \mathrm{mV}$, and reached a minimum value at $-400 \mathrm{mV}$. Polarization more negative than $-600 \mathrm{mV}$ led to the recovery of the length. At a potential regime more positive than $\phi=-400 \mathrm{mV}$, sulfate anions adsorb on the surface of gold. Gradual increase in the length from -400 to $500 \mathrm{mV}$ agreed with previously reported incre-

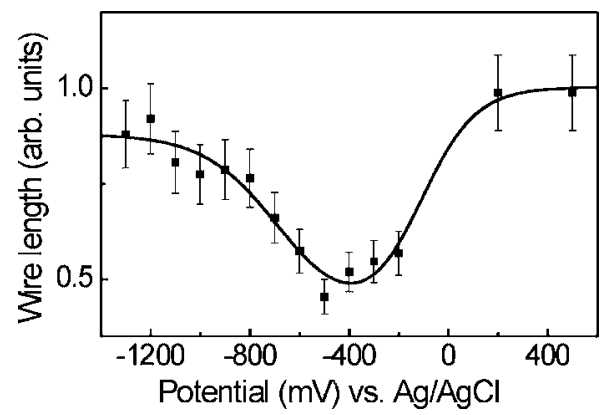

FIG. 3. Electrochemical potential dependence of the averaged stretch length of the gold monoatomic wire. 

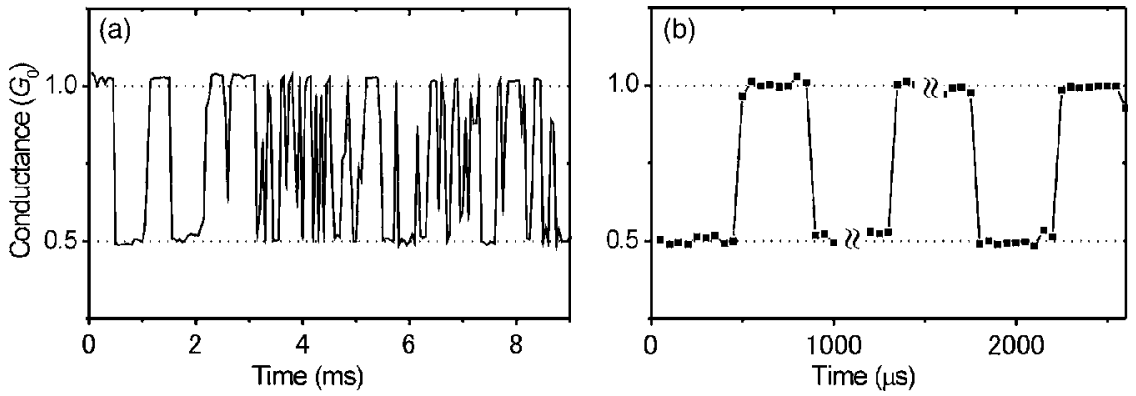

FIG. 4. Time-dependent change in the conductance of the gold nanowire at $\phi=-1000 \mathrm{mV}$. Data were acquired every $50 \mu \mathrm{s}$. ments in the amount of the adsorbed sulfate anions on the gold electrode. ${ }^{21}$ This result suggested that adsorption of the anions onto the gold nanowire led to stabilization of the gold monoatomic wire. Polarization to a potential more negative than $-400 \mathrm{mV}$ induces hydrogen evolution reaction at the gold electrode. ${ }^{22}$ Thus, recovery of the stretch length at the negative potential regime implied that evolved hydrogen molecules and/or adsorbed hydrogen could stabilize the gold monoatomic wire. These electrochemical potentialdependent changes in the stretch length were fully reversible. This fact implies that the stability of the gold monoatomic wire could be controlled by the electrochemical potential.

At the negative potential regime, reversible transition of the conductance between $1 G_{0}$ and $0.5-0.7 G_{0}$ was observed as shown in Fig. 1(c). Extended conductance traces shown in Fig. 4 proved that the changes between the two states occurred within $50 \mu \mathrm{s}$. This conductance fluctuation resulted in the fractional conductance peaks in the conductance histogram [see Fig. 1(f)]. The conductance fluctuated between two well-defined values, which strongly suggested the occurrence of a structural transition between two bistable states at the gold monoatomic wire. In solution, hydrogen evolution

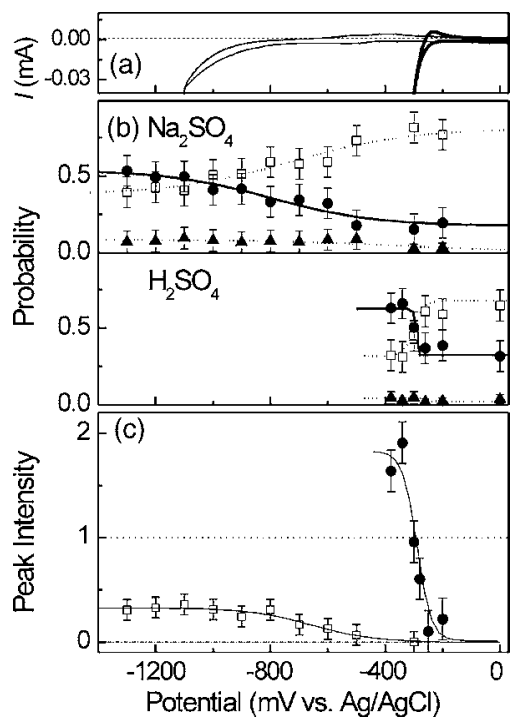

FIG. 5. (a) Voltammogram in $0.1 \mathrm{M} \mathrm{Na}_{2} \mathrm{SO}_{4}$ (thin line) and $50 \mathrm{mM} \mathrm{H}_{2} \mathrm{SO}_{4}$ (bold line). (b) The probability of each conductance trace in the presence of only fractional conductance step (triangle), only $1 G_{0}$ conductance step (box), and both steps (filled circle) as a function of electrochemical potential $(\phi)$. (c) The normalized fractional conductance intensity as a function of $\phi$ in $\mathrm{Na}_{2} \mathrm{SO}_{4}$ (box) and $\mathrm{H}_{2} \mathrm{SO}_{4}$ (filled circle). proceeds at the electrochemical potential in which the fractional value was observed. Therefore, hydrogen molecules and/or adsorbed hydrogen atoms may play a decisive role in the evolution of the fractional conductance.

Figure 5 shows potential dependences of the reductive current due to the hydrogen evolution together with those of the occurrence of the fractional values in $0.1 \mathrm{M} \mathrm{Na} \mathrm{SO}_{4}$. Figure 5(b) shows the probability of the conductance trace in the presence of only a fractional conductance step, only a $1 G_{0}$ conductance step, and both steps as a function of $\phi$. Figure 5(c) shows the intensity of the fractional conductance peak normalized by the $1 G_{0}$ peak. With an increase in current due to the hydrogen evolution reaction, the probability of the conductance trace with the fractional conductance steps and the intensity of the fractional conductance peak increased. In the presence of the fractional conductance, both $1 G_{0}$ and fractional conductance steps simultaneously appeared, suggesting that the fractional value originated from the conductance fluctuation. These results showed that evolved hydrogen induced the fractional conductance, which may reflect the occurrence of the transition between two bistable states of the gold monoatomic wire at the negative potential regime.

To confirm the effect of hydrogen on the fractional value, we studied electrochemical potential dependence of the conductance quantization behavior in acidic solution. Figure 6 shows the conductance histogram of gold nanowires observed at $\phi=+500$ and $-300 \mathrm{mV}$ in $50 \mathrm{mM} \mathrm{H}_{2} \mathrm{SO}_{4}$. The fractional conductance peak appeared at a potential regime more negative than $\phi=-200 \mathrm{mV}$. It is noteworthy that the

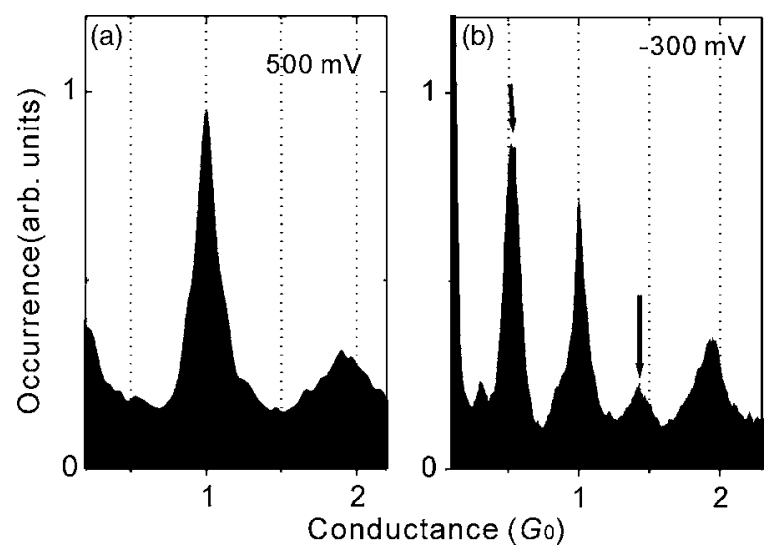

FIG. 6. Conductance histogram of gold nanowires in $50 \mathrm{mM}$ $\mathrm{H}_{2} \mathrm{SO}_{4}$ at electrochemical potential of (a) $+500 \mathrm{mV}$ and (b) $-300 \mathrm{mV}$. 
intensity of the fractional conductance peak was larger than that of the $1 G_{0}$ peak. We discuss this point later. In the solution with a lower $\mathrm{pH}\left(50 \mathrm{mM} \mathrm{H}_{2} \mathrm{SO}_{4}\right)$, the onset potential of the hydrogen evolution shifted to more positive potential than that which occurred in the solution with a neutral $\mathrm{pH}$ $\left(0.1 \mathrm{M} \mathrm{Na}_{2} \mathrm{SO}_{4}\right)$, as shown in Fig. 5(a). The occurrence of the fractional conductance shifted in accordance with that of the hydrogen evolution [see Fig. 5(c)]. Dependence of the solution $\mathrm{pH}$ confirmed that the fractional conductance observed in solution originated from the structure stabilized by hydrogen.

Recently, similar quantized conductance behavior was reported by Csonka et al. ${ }^{7}$ They observed a fractional conductance peak $\left(0.6 G_{0}\right)$ in a conductance histogram of gold nanowires under hydrogen dosing at $20 \mathrm{~K}$ in UHV, although the intensity of the fractional feature was very weak compared with that of the $1 G_{0}$ peak. They observed reversible conductance fluctuation between $1 G_{0}$ and fractional conductance in the conductance trace. They proposed that the fractional conductance value observed at the conductance fluctuation originated from the structural transition between an equal-spacing and dimerized wire, based on the following reasons. First, the fractional conductance peaks were not observed in $\mathrm{Cu}$ and $\mathrm{Ag}$, and $\mathrm{Au}$ forms a monoatomic wire, while $\mathrm{Cu}$ and $\mathrm{Ag}$ do not form a monoatomic wire. ${ }^{1}$ Second, a stretched single atom wire has a strong tendency to spontaneous dimerization-a predecessor for Peierls transition in extended 1D systems. ${ }^{23}$ Theoretical calculation supports that the conductance of a dimerized gold wire is $0.4-0.6 G_{0}{ }^{8}$ which is close to the observed values. Since conductance quantization behavior in UHV was similar to that observed in the present study, a similar phenomena would occur in both UHV and solution. The fractional conductance and conductance fluctuation observed in the present study might originate from the dimerized wire and the structural transition between equal spacing and the dimerized wire. If the dimerization is the origin of the fractional conductance, we could observe the Peierls transition of the 1D metal (monoatomic wire) at room temperature.

At the present stage, there is no direct proof of the reversible structural transition of the gold monoatomic wire in solution. As proposed by Csonka et al., the apparent effect of the hydrogen evolution reaction on the appearance of the fractional conductance value strongly suggests that adsorbed hydrogen molecules on the gold monoatomic wire could contribute to the formation of two bistable states of the monoatomic wire. Another possibility for the formation of the hydrogen incorporated wire can be considered, because a recent theoretical calculation showed that the incorporation of a hydrogen molecule in the gold monoatomic wire also decreased conductance below $1 G_{0},{ }^{9,10}$ Further investigation is needed to determine the structure with fractional conductance stabilized by hydrogen.

The present results clearly demonstrate the fabrication of a well-defined atomic structure with fractional conductance values controlled by the electrochemical potential. ${ }^{24}$ In contrast with the UHV case, we controlled the intensity of the fractional conductance peak (stability) by the electrochemical potential. Furthermore, the relative intensity of the fractional peak became stronger than that of the unit value (see Fig. 6). The specific structure with $0.5-0.7 G_{0}$ (fractional wire) became more stable than the structure with $1 G_{0}$ (normal wire). It should be noted that the intensity of the fractional conductance feature does not exceed the $1 G_{0}$ peak intensity even in the system in UHV at $20 \mathrm{~K},{ }^{7}$ that is, fractional wire cannot be kept as the most energetically favorable structure. The present observation proved that structural bistability of gold monoatomic wire could be tuned by changing the electrochemical potential.

Recently, several atomic quantum switches have attracted wide attention as a conceptual new electric switch. ${ }^{25}$ In the atomic quantum switch, a contacting metal atom is electrochemically deposited (on) or dissolved (off) in the contact area. On the other hand, in the present case, the conductance transition was based on the transition between two bistable structures, and thus, the movement of metal atom would be very small. Furthermore, both stability and structure of the monoatomic wire can be controlled at room temperature. Therefore, the present findings would also open an intriguing perspective for the emerging fields of quantum electronics and logics on the atomic scale in the future.

\section{CONCLUSIONS}

We have studied quantum conductance behavior of the gold nanowire under electrochemical potential control. A stable 1-nm-long monoatomic wire was formed in solution, and a certain atomic configuration of the wire with fractional conductance was stabilized by hydrogen. The conductance bistability of the gold monoatomic wire could be controlled by the electrochemical potential and the $\mathrm{pH}$ at room temperature.

\section{ACKNOWLEDGMENTS}

This work was partially supported by a Grant-in-Aid for Scientific Research A (No. 16205026) and Grant-in-Aid for Scientific Research on Priority Areas (Grant No. 17069001) from MEXT.
${ }^{1}$ N. Agrait, A. L. Yeyati, and J. M. van Ruitenbeek, Phys. Rep. 377, 81 (2003).

${ }^{2}$ J. C. Cuevas, A. Levy Yeyati, A. Martin-Rodero, G. R. Bollinger, C. Untiedt, and N. Agrait, Phys. Rev. Lett. 81, 2990 (1998).

${ }^{3}$ H. Ohnishi, Y. Kondo, and K. Takayanagi, Nature (London) 395,
780 (1998)

${ }^{4}$ A. I. Yanson, G. R. Bollinger, H. E. van den Brom, N. Agrait, and J. M. van Ruitenbeek, Nature (London) 395, 783 (1998).

${ }^{5}$ V. Rodrigues, J. Bettini, P. C. Silva, and D. Ugarte, Phys. Rev. Lett. 91, 096801 (2003). 
${ }^{6}$ N. Zabala, M. J. Puska, and R. M. Nieminen, Phys. Rev. Lett. 80, 3336 (1998).

${ }^{7}$ S. Csonka, A. Halbritter, G. Mihaly, E. Jurdik, O. I. Shklyarevskii, S. Speller, and H. van Kempen, Phys. Rev. Lett. 90, 116803 (2003).

${ }^{8}$ M. Okamoto and K. Takayanagi, Phys. Rev. B 60, 7808 (1999).

${ }^{9}$ R. N. Barnett, H. Hakkinen, A. G. Scherbakov, and U. Landman, Nano Lett. 4, 1845 (2004).

${ }^{10}$ N. V. Skorodumova and S. I. Simak, Phys. Rev. B 67, 121404(R) (2003).

${ }^{11}$ M. Brandbyge, K. W. Jacobsen, and J. K. Norskov, Phys. Rev. B 55, 2637 (1997).

${ }^{12}$ W. A. de Heer, S. Frank, and D. Ugarte, Z. Phys. B: Condens. Matter 104, 469 (1997).

${ }^{13}$ C. Z. Li, H. X. He, A. Bogozi, J. S. Bunch, and N. J. Tao, Appl. Phys. Lett. 76, 1333 (2000).

${ }^{14}$ A. L. Yeyati, A. Martin-Rodero, and F. Flores, Phys. Rev. B 56, 10369 (1997).

${ }^{15}$ C. Shu, C. Z. Li, H. X. He, A. Bogozi, J. Bunch, and N. J. Tao, Phys. Rev. Lett. 84, 5196 (2000).

${ }^{16}$ J. L. Li, T. Kanzaki, K. Murakoshi, and Y. Nakato, Appl. Phys.
Lett. 81, 123 (2002).

${ }^{17}$ M. Kiguchi, T. Konishi, and K. Murakoshi, Appl. Phys. Lett. 87, 43104 (2005).

${ }^{18}$ M. R. Sullivan, D. A. Boehm, D. A. Ateya, S. Z. Hua, and H. D. Chopra, Phys. Rev. B 71, 024412 (2005).

${ }^{19}$ M. Kiguchi, T. Konishi, and K. Murakoshi, Trans. Mater. Res. Soc. Jpn. 30, 1215 (2005).

${ }^{20}$ R. H. M. Smit, C. Untiedt, G. Rubio-Bollinger, R. C. Segers, and J. M. van Ruitenbeek, Phys. Rev. Lett. 91, 076805 (2003).

${ }^{21}$ H. Uchida, M. Hiei, and M. Watanabe, J. Electroanal. Chem. 452, 97 (1998).

${ }^{22}$ B. E. Conway and L. Bai, Electrochim. Acta 31, 1013 (1986).

${ }^{23}$ R. E. Peierls, Quantum Theory of Solids (Clarendon, Oxford, 1964).

${ }^{24}$ According to the recent reports [Sz. Csonka, A. Halbritter, and G. Mihaly, Phys. Rev. B 73, 075405 (2006)], a clear fractional peak was not observed in the conductance histogram by introducing hydrogen in UHV.

${ }^{25}$ F. Q. Xie, L. Nittler, Ch. Obermair, and Th. Schimmel, Phys. Rev. Lett. 93, 128303 (2004). 